\title{
AN EXTREMAL PROPERTY OF STOCHASTIC INTEGRALS
}

\author{
STEVEN ROSENCRANS
}

Abstract. In this paper we consider the stochastic integral $y_{t}=\int_{0}^{t} e(s, b) d b_{s}$ of a nonanticipating Brownian functional $e$ that is essentially bounded with respect to both time and the Brownian paths. Let $f$ be a convex function satisfying a certain mild growth condition. Then $E f\left(y_{t}\right) \leqq E f\left(\|e\| b_{t}\right)$, where $b_{t}$ is the position at time $t$ of the Brownian path $b$. As a corollary, sharp bounds are obtained on the moments of $y_{t}$. The key point in the proof is the use of a transformation, derived from Itô's lemma, that converts a hyperbolic partial differential equation into a parabolic one.

Let $(t, b) \rightarrow e(t, b)$ be a nonanticipating Brownian functional (see $[1])$ that is essentially bounded with respect to both the time $t(\geqq 0)$ and the Brownian paths $b$, i.e.,

$$
\|e\|:=\operatorname{essup}_{t, b}|e(t, b)|<\infty .
$$

Let $y_{t}$ be the stochastic (i.e., Itô) integral of $e$,

$$
y_{t}=\int_{0}^{t} e(s, b) d b_{s}, \quad t \geqq 0 .
$$

(By $b_{s}$ we mean the position of the path $b$ at time $s$, while $b$ denotes the entire path.)

Let $f$ be a convex function on $R$ satisfying the growth condition

$$
\text { (GC) } \quad|f(x)| \leqq K \exp (|x| a), \quad x \in R, \text { for some } a<2 \text {. }
$$

We consider the functional $e \rightarrow E f\left(y_{t}\right),\|e\| \leqq 1$, and prove that it attains its maximum value at the constant function $e \equiv 1$. $E$ is the expectation, i.e. integral over the space of Brownian paths with Wiener measure. As a corollary we obtain new and sharp bounds on the moments of $y_{t}$. The essential ingredient in the proof is the use of Itô's lemma to convert a hyperbolic equation or inequality for a function $h(x, t)$ into a parabolic equation or inequality for $H(x, t):=E h\left(x, y_{t}\right)$. This last point is discussed further in Remark 2, where references are given to related work.

Received by the editors June 22, 1970.

AMS 1970 subject classifications. Primary 60H05; Secondary 35K15, 35L15.

Key words and phrases. Nonanticipating Brownian functional, stochastic integral, Itô's lemma, Bessel process, moments, parabolic equation, hyperbolic equation. 
THEOREM. Let $f$ be a convex function on $R$ that satisfies (GC). Let $e$ be a nonanticipating Brownian functional with $\|e\|<\infty$ and let $y_{t}(i \geqq 0)$ be the stochastic integral of $e$. Then for $t \geqq 0, E f\left(y_{t}\right) \leqq E f\left(\|e\| b_{t}\right)$.

Proof. Let

$$
\begin{aligned}
h(x, t) & =C^{-1} \int_{-1}^{1} f(t+x m)\left(1-m^{2}\right)^{(n-3) / 2} d m, \\
C & =\int_{-1}^{1}\left(1-m^{2}\right)^{(n-3) / 2} d m .
\end{aligned}
$$

Then $h$ is continuous in $D=\{(x, t) \mid x \geqq 0,-\infty<t<\infty\}$, and from

$$
h(x, t)=C^{-1} x^{-1} \int_{t-x}^{t+x} f(w)\left\{1-\left(\frac{w-t}{x}\right)^{2}\right\}^{(n-3) / 2} d w,
$$

it follows that for odd $n, h \in C^{(n-3) / 2}\left(D^{0}\right)$, while for even $n$, $h \in C^{(n-2) / 2}\left(D^{0}\right)$. In particular if $n \geqq 7, h \in C^{2}\left(D^{0}\right)$. Let us assume from now on that $n$ is so chosen. From (1) and (2) one can verify that $h$ is a solution to the following boundary value problem for the Darboux equation (see $[2$, p. 700$]$ ):

$$
\begin{aligned}
h_{t t} & =G h, \quad(x, t) \in D^{0}, \\
h(0, t) & =f(t),
\end{aligned}
$$

where $G$ is the operator $G h:=h_{x x}+((n-1) / x) h_{x}$.

We note the estimate

$$
P\left\{\left|y_{t}\right|>z\right\} \leqq 2 \exp \left\{\frac{-z^{2}}{2\|e\|^{2} t}\right\}
$$

which is contained in $[1$, p. 25]. Owing to this bound and to the fact that the function $t \rightarrow h(x, t)$ satisfies (GC) it is possible to define the "transform"

$$
H(x, t):=E h\left(x, y_{t}\right)
$$

which is then a continuous function in $A=\{(x, t) \mid x \geqq 0, t \geqq 0\}$. Next we compute the stochastic differential of $h\left(x, y_{t}\right)$, toward showing that $H$ satisfies a certain parabolic inequality. Thus

$$
d h=h_{t} d y_{t}+\frac{1}{2} h_{t t}\left(d y_{t}\right)^{2}=h_{t} e d b_{t}+\frac{1}{2} h_{t t} e^{2} d t .
$$

We claim that, for any $s>0$,

$$
E \int_{0}^{s} h_{t}^{2}\left(x, y_{r}\right) d r<\infty, \quad x>0 .
$$


To see this, we compute $h_{t}$, from (2), with the result

$$
h_{t}(x, t)=(n-3) x^{-1} \int_{-1}^{1} f(t+x m) m\left(1-m^{2}\right)^{(n-5) / 2} d m .
$$

From this representation (7) follows after a short calculation using (GC), and (4), and Schwarz' inequality. But (7) implies that in (6) the "Brownian" term $h_{t} e d b_{t}$ has mean zero, so $t \rightarrow H(x, t)$ is a smooth function (for $x>0$ ) and

$$
H_{\imath}=\frac{1}{2} E\left[e^{2} h_{\iota t}\right] .
$$

Now it is immediate from (1) that $t \rightarrow h(x, t)$ is convex. Since $h \in C^{2}\left(D_{0}\right)$ it follows that $h_{t t} \geqq 0$. This and (8) imply

$$
\begin{aligned}
H_{t} & \leqq \frac{1}{2}\|e\|^{2} E\left(h_{t t}\right)=\frac{1}{2}\|e\|^{2} E(G h) \\
& =\frac{1}{2}\|e\|^{2} G(E h)=\frac{1}{2}\|e\|^{2} G H \quad \text { for }(x, t) \in A^{0} .
\end{aligned}
$$

This is the parabolic inequality that we referred to before.

We are now finished with the first part of the proof. The second part of the proof consists of randomizing the space coordinate of $H$, just as in the first part we randomized the time coordinate of $h$.

We introduce the diffusion $\left\{x_{t}, t \geqq 0\right\}$ governed by the operator $\|e\|^{2} G / 2$ and starting at the origin. Actually there are two such diffusions, one positive and one negative. We take the positive one, which is the $n$-dimensional Bessel process with a certain scaling. I.e., $x_{t}$ is the solution to the stochastic differential equation

$$
d x_{t}=\frac{(n-1)\|e\|^{2}}{2 x_{t}} d t+\|e\| d b_{t}^{*}, \quad x_{0}=0, \quad x_{t} \geqq 0 \quad \text { for } t>0,
$$

where $\left\{b_{t}^{*}, t \geqq 0\right\}$ is a Brownian motion independent of $\left\{b_{t}, t \geqq 0\right\}$. (See $[1$, p. 18, p. 47, p. 80$]$ for information about the Bessel process.) We need the fact that (almost surely) this process does not touch zero for positive times. Because of this, if $\epsilon$ and $s, s>\epsilon>0$ are two fixed numbers, we have $\left(x_{t}, s-t\right) \in A^{0}$ for $\epsilon<t<s$. This permits the application of Itô's lemma for the computation of the stochastic differential of $H\left(x_{t}, s-t\right)$ for $\epsilon<t<s$ :

$$
\begin{aligned}
d H\left(x_{t}, s-\right. & t)=H_{x} d x_{t}+\frac{1}{2} H_{x x}\left(d x_{t}\right)^{2}-H_{t} d t \\
= & H_{x}\left(\frac{(n-1)\|e\|^{2}}{2 x_{t}} d t+\|e\| d b_{t}^{*}\right)+\frac{1}{2} H_{x x}\|e\|^{2} d t-H_{t} d t \\
= & \left(\frac{1}{2}\|e\|^{2} G H-H_{t}\right) d t+H_{x}\|e\| d b_{t}^{*} .
\end{aligned}
$$

We claim that 


$$
E \int_{\epsilon}^{s} H_{x}^{2}\left(x_{t}, s-t\right) d t<\infty .
$$

To see this, note $H_{x}^{2}(x, t)=\left(E h_{x}\left(x, y_{t}\right)\right)^{2} \leqq E h_{x}^{2}\left(x, y_{t}\right)$, so it is sufficient to show

$$
E \int_{t}^{s} h_{x}^{2}\left(x_{t}, y_{s-t}\right) d t<\infty
$$

From (2) we find

$$
h_{x}(x, t)=x^{-1} \int_{-1}^{-1} f(t+x m)\left(1-m^{2}\right)^{(n-5) / 2}\left((n-2) m^{2}-1\right) d m,
$$

so

$$
h_{x}^{2}(x, t) \leqq \text { const } x^{-2} \int_{-1}^{-1} f^{2}(t+x m) d m \leqq \text { const } x^{-2} \exp \left[(|t|+x)^{a}\right],
$$

i.e.

$$
h_{x}^{2}\left(x_{t}, y_{s-t}\right) \leqq \text { const } \overline{x_{t}^{-2}} \exp \left[\left(\left|y_{s-t}\right|+x_{t}\right)^{a}\right]
$$

Thus

$$
E h_{x}^{2}\left(x_{t}, y_{s-t}\right) \leqq \text { const } E^{1 / 2}\left[x_{t}^{-4}\right] E^{1 / 2}\left[\exp \left[2\left(\left|y_{s-t}\right|+x_{t}\right)^{a}\right]\right] .
$$

Now we use the distribution (see e.g. $[3$, p. 60])

(13) $P\left\{x_{t} \in d z\right\}=\left.(2 \pi t)^{-n / 2} \exp \left[-z^{2} / 2 t\|e\| \|^{2}\right] z^{n-1}\|e\|\right|^{1-n} d z, \quad z \geqq 0$.

Since $n>4, E x_{t}^{-4}$ is a continuous and bounded function of $t$ for $t \geqq \epsilon$. The second term in (12) is treated just as before, using also (13) now.

We now return to (10) and note that (11) implies that the mean of the "Brownian" term $H_{x}\|e\| d b_{t}^{*}$ is zero, so

$$
\frac{d}{d t} E H\left(x_{t}, s-t\right)=E\left\{\frac{1}{2}\|e\|^{2} G H-H_{t}\right\} \geqq 0 \quad \text { for } \epsilon<t<s
$$

by (9).

Thus successively setting $t=\epsilon$ and $t=s$, we find

$$
E H\left(x_{\epsilon}, s-\epsilon\right) \leqq E H\left(x_{s}, 0\right) .
$$

Next note that in the case $e \equiv\|e\|$ the parabolic inequality (9) is replaced by an equality. Thus if we define

$$
\tilde{H}(x, t)=E h\left(x,\|e\| b_{t}\right),
$$


we have, corresponding to (15),

$$
E \tilde{H}\left(x_{\epsilon}, s-\epsilon\right)=E \tilde{H}\left(x_{s}, 0\right) .
$$

But, from the definitions of $H$ and $\tilde{H}$,

$$
E \tilde{H}\left(x_{8}, 0\right)=E H\left(x_{s}, 0\right),
$$

so from (15), (17), and (18), $E H\left(x_{\epsilon}, s-\epsilon\right) \leqq E \tilde{H}\left(x_{\epsilon}, s-\epsilon\right)$. This is true for all $\epsilon$ satisfying $0<\epsilon<s$. By the continuity of $H$ and $\tilde{H}, E H(0, s)$ $\leqq E \tilde{H}(0, s)$. From (3), (5), and (16), this is just $E f\left(y_{8}\right) \leqq E f\left(\|e\| b_{s}\right)$. Q.E.D.

Corollary. For $p \geqq 1$,

$$
E\left|\int_{0}^{t} e d b\right|^{p} \leqq\|e\|{ }^{p} E\left|b_{t}\right|^{p} .
$$

Proof. Take $f(x)=|x|^{p}$.

REMARK 1. These bounds are clearly sharp, since they are attained in the case of a constant function $e$. For $p=4$, the Corollary states $E\left(\int e d b\right)^{4} \leqq 3\|e\|^{4} t^{2}$ which should be compared with the estimate of Skorokhod [1, p. 40] in which the constant 3 is replaced by 36 , and with that of Zakai [4] in which 3 is replaced by 9; but note that the latter estimates are actually special cases of estimates that these authors have given for a wider class of functions.

REMARK 2. We noted in the proof of the Theorem that in the case $e \equiv$ constant, (9) becomes an equality rather than an inequality. It is clear that the same result holds true for any linear operator $G$ independent of time. ( $G$ need not be a differential operator.) That is, if $h$ is a smooth solution to the equation $h_{t t}=G h$ and if the function $t$ $\rightarrow h_{t}(x, t)$ satisfies (GC) then the transform $H(x, t)=E h\left(x, 2^{1 / 2} b_{t}\right)$ satisfies the equation $H_{t}=G H$. Moreover both $h$ and $H$ satisfy the same initial condition, i.e., $H(x, 0)=h(x, 0)$.

(The simplest application of this fact is to the operator $G=\partial^{2} / \partial x^{2}$. In this case the Poisson integral solution to the heat equation is derived as a consequence of the d'Alembert solution to the wave equation. These relationships have been noted previously (see [5][9]), but the present proof is different.)

REMARK 3. The idea of differentiating the solution of a parabolic inequality along its "characteristic" diffusion $x_{s}$ was introduced in [10].

Remark 4. Mark Pinsky has shown the author a simple proof of the Theorem in the special case of exponential functions (and hence superpositions of these). Consider the exponential martingale 


$$
\eta=\exp \left(\theta \int e d b-\theta^{2} / 2 \int e^{2} d s\right)
$$

Since $E_{\eta} \equiv 1$, a simple estimate proves our result for the special case $f(x)=\exp (\theta x)$.

Acknowledgment. This research was supported in part by NSF Contract GP-12722. The author would like to thank E. D. Conway and J. A. Goldstein for several helpful conversations.

\section{BIBLIOGRAPHY}

1. H. P. McKean, Jr., Stochastic integrals, Probability and Math. Statist., no. 5, Academic Press, New York, 1969. MR 40 \#947.

2. R. Courant, Methods of mathematical physics. Vol. 2: Partial differential equations, Interscience, New York, 1962. MR 25 \#4216.

3. K. Ito and H. P. McKean, Jr., Diffusion processes and their sample paths, Die Grundlehren der math. Wissenschaften, Band 125, Academic Press, New York; Springer-Verlag, Berlin, 1965. MR 33 \#8031.

4. M. Zakai, Some moment inequalities for stochastic integrals and for solutions of stochastic differential equations, Israel J. Math. 5 (1967), 170-176. MR 37 \#991.

5. R. Hersh, Explicit solution of a class of higher-order abstract Cauchy problems (to appear).

6. L. R. Bragg and J. W. Dettman, Related problems in partial differential equations, Bull. Amer. Math. Soc. 74 (1968), 375-378. MR 37 \#560.

7. N. P. Romanov, On one-parameter groups of linear transformations. I, Ann. of Math. (2) 48 (1947), 216-233. MR 8, 520.

8. R. Hersh and R. Griego, Random evolutions-theory and applications (to appear).

9. A. V. Balakrishnan, Abstract Cauchy problems of the elliptic type, Bull. Amer. Math. Soc. 64 (1958), 290-291. MR 21 \#4294.

10. E. D. Conway, Stochastic characteristics for parabolic equations, Tulane University (multilithed).

Tulane University, New Orleans, Louisiana 70118 